\title{
Métodos de avaliação de IHC no contexto de Jogos Sérios Educacionais: Um Mapeamento Sistemático
}

\author{
Taynara Cerigueli Dutra ${ }^{1}$, Diego Fellipe Tondorf ${ }^{1}$, Thiago A. Zils ${ }^{1}$, \\ André E. G. Ferreira ${ }^{1}$, Isabela Gasparini ${ }^{1}$, \\ Marcelo da Silva Hounsell ${ }^{1}$, Eleandro Maschio $^{2}$ \\ ${ }^{1}$ Universidade do Estado de Santa Catarina (UDESC)) Joinville - SC - Brasil \\ ${ }^{2}$ Universidade Tecnológica Federal do Paraná (UTFPR) Guarapuava - PR - Brasil \\ \{taynara.dutra, diego.tondorf, andre.ferreira\}@edu.udesc.br \\ \{isabela.gasparini, marcelo.hounsell\}@udesc.br, \\ eleandromeutfpr.edu.br
}

\begin{abstract}
This work presents a Systematic Literature Mapping to identify how educational serious digital games have been evaluated, by verifying evaluation methods, criteria, context, and types of games used. A total of 1221 papers were obtained, which after applying objective and subjective filters, resulted in 48 selected papers. It was found that the majority used investigation methods such as questionnaires, to verify elements such as learning, motivation, and user experience. Furthermore, regarding the subjects, the vast majority aims at evaluating games with elementary school students. Finally, as for control devices, there are similarities between mouse, keyboard, and touchscreen.
\end{abstract}

Resumo. Este trabalho apresenta um Mapeamento Sistemático da Literatura realizado para identificar como os jogos digitais sérios educacionais têm sido avaliados, verificando os métodos e critérios de avaliação, o contexto e os tipos de jogos usados. Foram obtidos 1.221 artigos que, após a aplicação de filtros objetivos e subjetivos, resultaram em 48 artigos selecionados. Constatou-se que a maioria são métodos de investigação, como questionários, para verificar elementos como aprendizagem, motivação e experiência do usuário. Além disso, em relação ao contexto, a grande maioria visa avaliar jogos com estudantes do ensino fundamental. Por fim, quanto aos dispositivos de controle, existem semelhanças entre mouse, teclado e tela sensível ao toque.

\section{Introdução}

O uso de jogos de computador despertou interesse entre pesquisadores para estudos da aplicabilidade desses jogos como uma abordagem para o ensino-aprendizagem de conteúdos regulares. Jogos digitais promovem diferentes emoções com base nas experiências e situações que propiciam aos jogadores, como entusiasmo, diversão, desafios, frustração e motivação [Ramos et al. 2020]. Além do mais, favorecem o desenvolvimento de habilidades cognitivas e podem ser utilizados nos mais diversos contextos.

Os jogos (digitais) com objetivos específicos, intencionais e prévios são nomeados de Jogos Sérios (JS). Os JS são jogos em que o objetivo primário não é divertir, mas sim aprimorar o interesse pela aprendizagem [Prensky 2003]. Os gêneros dos JS podem ser 
variados, desde que o intuito seja instruir algum conteúdo específico, podendo auxiliar nas mais diversas áreas, como na saúde, na formação militar, na educação colaborativa, na formação profissional e entre outras [Derryberry 2007].

Os JS podem ser aplicados aos mais diversos segmentos e um deles é a educação. Os JS proporcionam alterações positivas e inovadoras ao ensino da atual geração de estudantes [Anastasiadis et al. 2018]. Assim, jogos digitais com fins educativos são um subgrupo dos JS, sendo classificados como Jogos Digitais Educacionais (JDEs). Os JDEs aplicados a contextos educacionais precisam conter objetivos pedagógicos bem definidos, seguindo uma metodologia como orientação do processo [Prieto et al. 2005]. Também, são eficientes ferramentas instrucionais devido às suas características, pois possibilitam divertir e engajar, enquanto facilitam o aprendizado, aumentam a capacidade de retenção do conhecimento adquirido e exercitam funções mentais e intelectuais dos jogadores. Portanto, as novas tecnologias inseridas nas salas de aula tornaram-se essenciais, não somente devido aos seus benefícios, mas também por atenderem as atuais necessidades sociais e culturais da sociedade [Tarouco et al. 2004].

O uso de JDEs muda o paradigma de ensino: do aprender ouvindo (learn by listen) para o aprender fazendo (learning by doing). Além de que, permite aumentar o interesse dos estudantes em assuntos abordados em aula, fornecendo práticas dinâmicas e lúdicas sobre os mais diversos conteúdos [Peixoto et al. 2014]. Entretanto, a adesão a esses jogos ainda é moderada [Grove et al. 2012], motivada pela falta de avaliações bem fundamentadas em relação ao propósito principal, que é ensinar [Cascini e Campos 2015].

O presente trabalho objetiva a realização de um Mapeamento Sistemático da Literatura (MSL) com a finalidade de identificar como os JDEs estão sendo avaliados nos últimos anos.

\section{Trabalhos relacionados}

Cascini e Campos (2015) apresentaram o crescente interesse em JDEs e a necessidade dos métodos de avaliação para medir o benefício desses jogos. Com o intuito de identificar como jogos educacionais multiusuários são avaliados, os autores realizaram uma revisão sistemática da literatura. Como resultado, contatou-se que o método de avaliação mais utilizado é o questionário e que outros métodos utilizados são observação, testes e entrevistas. Em relação ao que é avaliado, a maioria considera a interação, o aprendizado e a experiência do usuário. Sobre a efetividade dos testes, são notáveis os resultados positivos da utilização dessas técnicas. Por fim, os autores apontam que, na maioria dos casos, a avaliação está restrita apenas a testes pilotos ou avaliações subjetivas. Também, dispõem um conjunto de questões para auxiliar a avaliação de um jogo.

Wangenheim et al. (2017) realizaram uma revisão sistemática da literatura para verificar e mapear heurísticas de avaliação de usabilidade para JDEs. Com base nos artigos obtidos, foram encontradas 7 heurísticas usadas na avaliação de jogos educacionais, em que a maioria são heurísticas adaptadas de outras, principalmente de Nielsen (1994), aplicadas pelos usuários finais e especialistas. Ademais, grande parte das avaliações não apresentaram uma metodologia clara da realização da avaliação, nem como as heurísticas foram criadas e validadas.

Esses trabalhos demonstram a importância dos jogos ao ambiente educacional e devido ao seu objetivo primário e sério, as etapas de avaliação e validação são essenciais 
para garantir a qualidade do jogo final. Entretanto, esses trabalhos não apresentam os métodos utilizados e os critérios avaliados especificadamente para JDEs. À vista disso, para averiguar como os JDEs são avaliados atualmente, foi realizado um MSL com o intuito de identificar dados quantitativos a respeito das avaliações desses jogos. Com as informações angariadas, pretende-se compreender como realizar uma efetiva avaliação de um JDE, bem como, quais as melhores métricas usadas para essa avaliação e o públicoalvo.

\section{Metodologia}

O MSL relatado no presente trabalho segue o processo descrito por Petersen et al. (2008).

\subsection{Questões de pesquisa}

O objetivo principal deste estudo é quantificar e descrever sobre a avaliação de JDEs e para isso estabeleceu-se a seguinte Questão Primária (QP) para o mapeamento: Como os jogos digitais sérios educacionais são avaliados?

Para auxiliar o compreendimento sobre o tema e organização dos resultados, as seguintes Questões Secundárias (QS) foram estabelecidas: (QS1) Quais métodos são utilizados nas avaliações? (QS2) Quais critérios são avaliados? (QS3) Quem avalia os jogos sérios? (QS4) Em que contexto são avaliados? (QS5) Para qual método de controle o jogo é desenvolvido?

\subsection{Definições de busca}

Os trabalhos foram buscados por meio de uma string de busca, que foi definida para que os resultados se enquadrassem com a temática dos jogos digitais sérios/educacionais. A pesquisa foi realizada e obtiveram-se os artigos que atendiam a seguinte string:

(evaluation OR assessment OR test) AND (model OR method OR framework OR scale) AND (serious OR educati* OR appl*) AND (game* AND digital)

Como Mecanismo de Busca Acadêmica (MBA) foram utilizados o Web of Science (WOS), a ACM Digital Library e o IEEE Xplore, por terem maior quantidade de recursos disponíveis [Buchinger et al. 2014] e pela proximidade das bases com a área da Computação.

A pesquisa, executada entre os meses de maio e junho de 2021, resultou em 1221 artigos ao total. A quantidade respectiva por cada base e cada conjunto de etapas pode ser visualizada na Tabela 1. A base Web of Science engloba as mais diversas áreas da ciência, portanto com o propósito de buscar resultados apenas relacionados a área da Computação e Educação, empregou-se o filtro por categorias.

\subsection{Seleção dos trabalhos}

Para a seleção dos trabalhos, foram definidos os Critérios Objetivos (CO) e os critérios subjetivos, que se subdividem em Critérios de Exclusão (CE) e Critérios de Inclusão (CI). Em análises iniciais, conforme pode ser visualizado na Figura 1, contatou-se que a partir do ano de 2014, houve um aumento significativo nos trabalhos de avaliação de JDEs. Dessa forma, instituiu-se que esse seria o ano inicial para filtragem dos artigos. 
Como Critérios Objetivos (CO) para seleção dos artigos, foram estabelecidos: (1)Possuir acesso pela CAPES ou gratuito na web; (2) Ser escrito em inglês ou em português; (3) Ser artigo científico de periódicos e eventos; (4) Artigos entre 2014 e 2021; (5) Ser artigo completo (possuir quatro ou mais páginas); (6) Ser artigo primário; e (7)Artigos não duplicados.

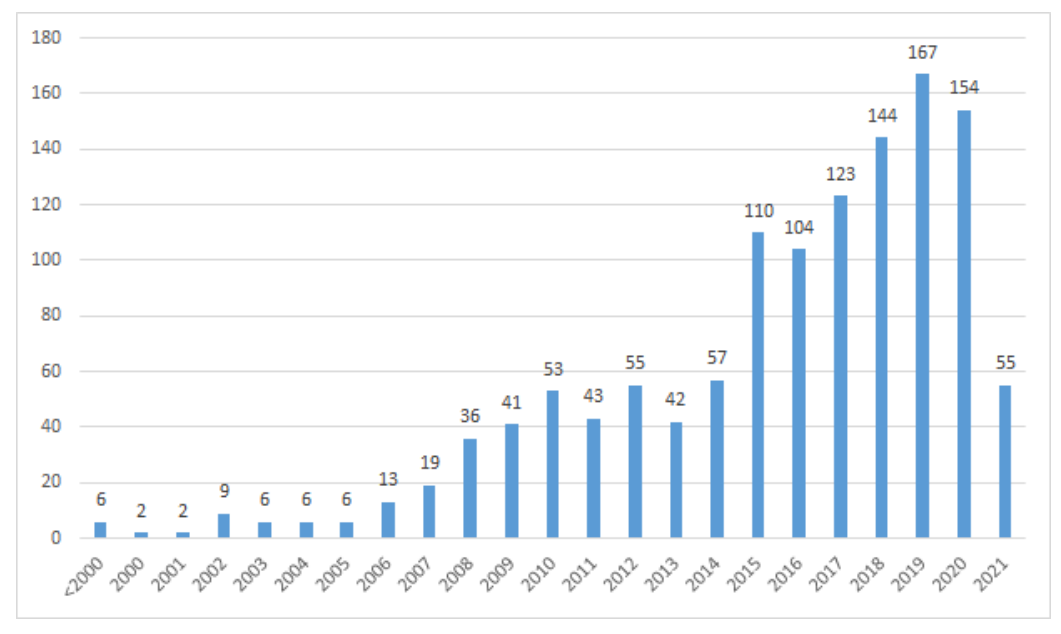

Figura 1. Publicações por ano de artigos sobre avaliação de JDEs

Para cada MBA, foram utilizadas as ferramentas disponíveis para filtragem dos dados de ano, linguagem e tipo de publicação, por exemplo. Essa filtragem automática foi denominada Critérios Objetivos Possíveis (COPs). Para os demais COs, que não puderam ser verificados diretamente no MBA, foi realizada a verificação manual, selecionando/removendo os artigos conforme COs citados anteriormente.

Os CEs, dispostos a seguir, foram estabelecidos para a remoção de trabalhos não pertencentes ao tema e para que os resultados filtrados e incluídos pelo CI sejam utilizados para responder as questões de pesquisa. Durante a análise, os trabalhos que atendiam ao menos um dos CEs foram removidos: CE1. Não ser jogo digital; CE2. Não ser jogo da área educacional; CE3. Apresentar métodos de avaliação sem testes; CE4. Jogos que não foram avaliados; (CE5. Ser jogo 3D.

Foi definido um CI, para os artigos que passassem por todos os CEs: CI1. Artigos que avaliem jogos sérios educacionais e apresentem as formas de avaliação.

Tabela 1. Quantidade de artigos retornados por cada MBA

\begin{tabular}{cccccc}
\hline Mecanismo de Busca & String Base & COP & CO1-7 & CE1-5 & CI1 \\
\hline Web of Science & 754 & 389 & 248 & 38 & 30 \\
IEEE & 366 & 217 & 191 & 14 & 14 \\
ACM & 101 & 44 & 40 & 4 & 4 \\
\hline Total & 1221 & 650 & 479 & 56 & 48 \\
\hline
\end{tabular}

Para a verificação de todos os critérios (COs, CEs e CI) executou-se, em 650 artigos, a análise do título, resumo, palavras-chave, e o artigo completo até passar por todos os critérios. Ao haver dúvidas a respeito da aplicação dos critérios, foi deliberado 
entre os autores e estabelecido um consenso para o aceite ou não do artigo. Os trabalhos que satisfizeram todos os COs, CEs e o CI foram aceitos para a extração dos dados. A Tabela 1 apresenta os quantitativos de cada etapa.

\subsection{Extração e classificação dos dados}

A extração dos dados seguiu o processo: leu-se as informações no resumo, caso não fosse viável obter os dados necessários, outras partes do artigo foram verificadas, como a introdução, conclusão e por fim, todo o trabalho [Petersen et al. 2008].

Destaca-se que quatro pesquisadores realizaram todo o processo de análise e extração e em uma segunda revisão, leram todos os trabalhos aceitos - o que fornece maior garantia da integridade dos resultados frente ao objetivo do presente trabalho. Outros três pesquisadores atuaram conferindo o protocolo, definindo os objetivos e critérios, revisando o texto e as interpretações. Os dados resultantes das análises foram tabulados e organizados em uma planilha eletrônica, para facilitar a visualização dos dados por filtros ou comandos, como a validação de duplicatas, por exemplo. Todas as análises e classificações realizadas estão dispostas na Tabela disponível no link ${ }^{1}$.

\section{Análise e Resultados}

Ao final, 48 artigos foram aceitos. Eles apresentavam a avaliação de JDEs e incluíam informações a respeito dos métodos adotados e o processo realizado. Esses artigos estão disponíveis ${ }^{2}$ e serão referenciados pelo número de identificação estabelecido.

\subsection{Métodos de avaliação dos JDEs}

A avaliação de Interação Humano-Computador (IHC) de sistemas computacionais visa coletar dados para determinar como os usuários finais devem utilizar o produto, em uma determina tarefa, em um certo ambiente. Assim, os métodos de avaliação podem ser agrupados em investigação, inspeção e observação [Barbosa et al. 2021]. Com base nessa classificação, os artigos foram analisados e, conforme os métodos de avaliação apresentados, foram inseridos em um ou mais grupos. A Figura 2 apresenta o gráfico com a quantidade de artigos pertencentes a cada um desses grupos. Destaca-se que, em vários artigos, utilizou-se mais de um método de avaliação e portanto um trabalho pode estar contido em mais de um grupo. A grande maioria dos artigos utilizam métodos do grupo de investigação, principalmente os questionários, mas também observação, tendo como método o estudo de campo.

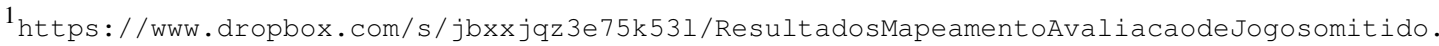
xlsx?dl=0 - Guias: Classificação por Avaliação, Classificação por Pessoas e Classificação por Jogos.

${ }^{2}$ Guia: Lista de Artigos Aceitos (no mesmo link anterior).
} 


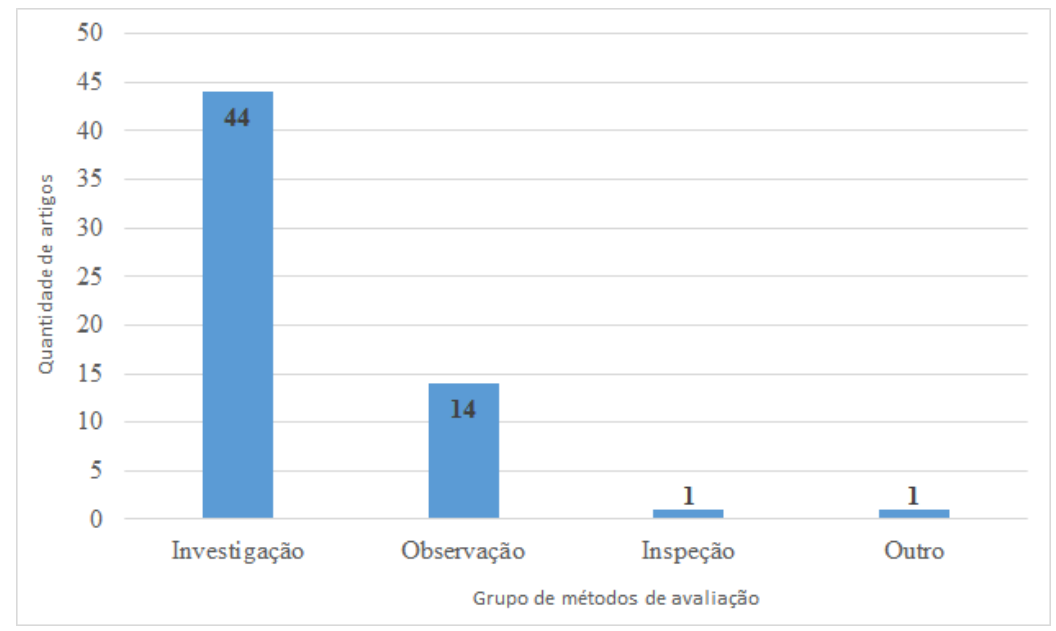

Figura 2. Grupos dos Métodos de Avaliação

Conforme apresentado na Figura 3, os questionários possuem uma ampla utilização para a avaliação dos jogos, o que corrobora com a afirmação de Barbosa et al. (2021), que os questionários são uma das técnicas de coleta de dados mais utilizadas. Isso ocorre devido à sua facilidade de uso para a obtenção dos mais diversos dados e a possibilidade de aplicação a inúmeros contextos. Além de que, é possível angariar informações de um grande número de pessoas, podendo essas estar geograficamente dispersas. Também por intermédio dos questionários, é possível a disponibilização de perguntas abertas e fechadas. Entretanto, geralmente se utiliza de questões fechadas para facilitar o preenchimento por parte do avaliado e também a análise quantitativa das informações por parte do avaliador [Barbosa et al. 2021]. Com a análise dos grupos de métodos e dos métodos verificados, foi possível responder a QS1.

De acordo com as análises realizadas, os questionários foram empregados para a verificação da aprendizagem com os pré e pós testes (ex: artigos 98, 118, 127, 227, etc.), para avaliação da usabilidade (ex: artigos 8, 170, 575, etc.), motivação (ex: artigos 128, 178, 227, etc), experiência de usuário (ex: artigos 269, 300, 430, etc.), dentre outros. Esses questionários, em muitos trabalhos foram criados pelos próprios avaliadores (ex: artigos 128, 178, 227, 391, etc.). Em outros, utilizaram-se modelos presentes na literatura, como é o caso de Costa et al. (2018), que utilizou o modelo de questionário Self-Assessment Manikin (SAM) (artigo 82), o qual se refere a um questionário baseado em imagens desenvolvido para medir respostas emocionais. Outro exemplo é o trabalho Yuliana et al. (2019), que realiza a avaliação da usabilidade do jogo educativo com o System Usability Scale (artigo 170).

Os artigos foram verificados e classificados conforme o tipo de dado extraído, sendo 45 trabalhos quantitativos e 12 qualitativos. Destaca-se que, devido às avaliações utilizarem mais de um método, um único trabalho pode estar quantificado como informações quantitativas e qualitativas.

As avaliações dos JDEs eram realizadas com o objetivo de validar algum aspecto dos jogos, como a capacidade de promover a aprendizagem, motivação, engajamento nos estudantes ou então, a usabilidade do jogo em si, a satisfação em utilizar a ferramenta para aprender, entre outros objetivos. Com isso, é possível responder à questão secundária QS2, identificando quais os critérios avaliados em JDEs. Os trabalhos obtidos foram 


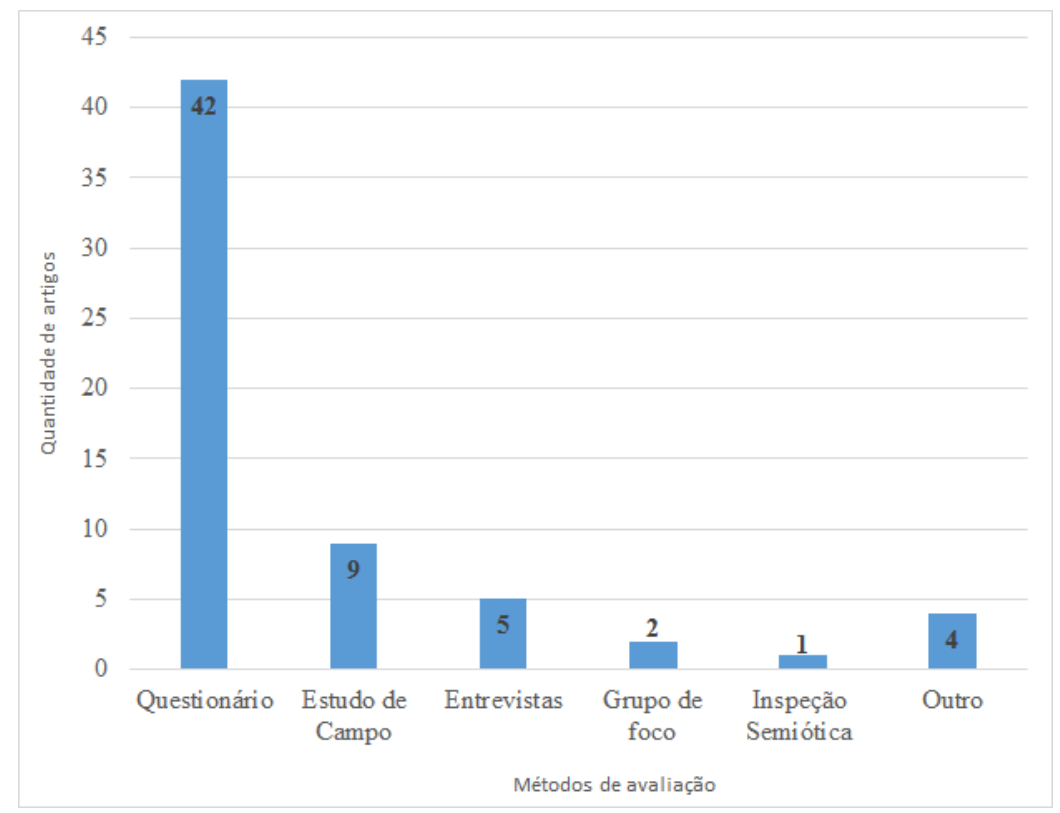

Figura 3. Métodos de Avaliação

classificados conforme os objetivos com maior ocorrência, conforme pode ser visualizado na Figura 4. Entretanto, constatou-se outros critérios, como exemplo, a afetividade, a compaixão, a ansiedade, a carga cognitiva e devido a diversidade, estes foram classificados como "Outros" e podem ser visualizados no seguinte link ${ }^{3}$. Deve-se considerar que os objetivos foram classificados conforme descritos pelos autores dos trabalhos avaliados, não entrando no mérito de que alguns conceitos podem englobar outros, como é o caso da experiência do usuário estar relacionada também, a motivação e ao engajamento.

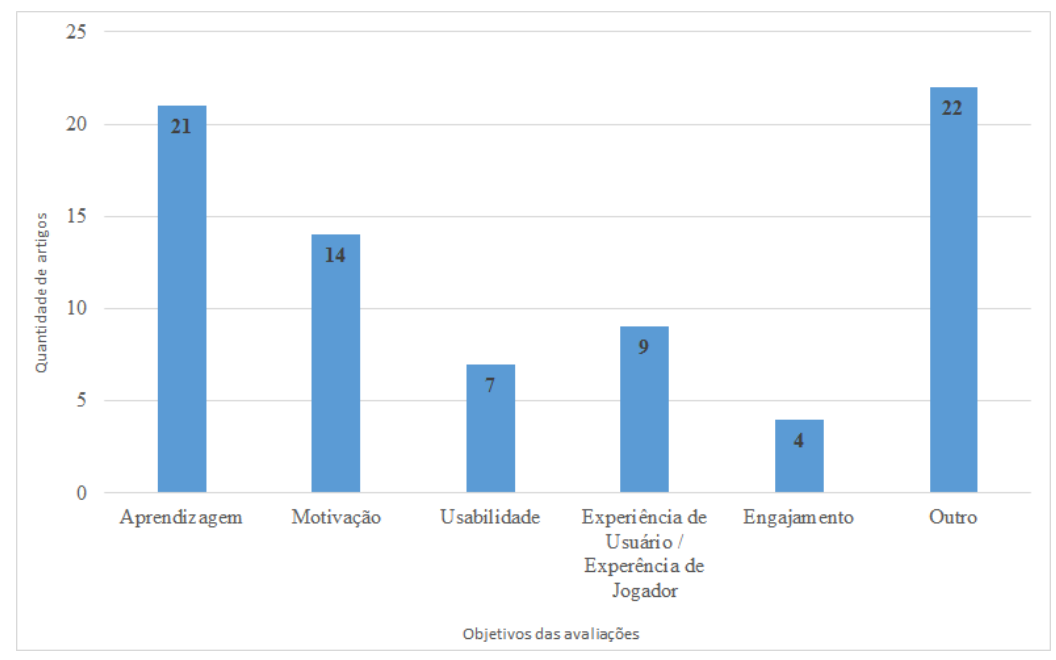

Figura 4. Objetivos de Avaliação

Os métodos de avaliação de IHC também podem ser classificados como métodos de avaliação formativos e métodos de avaliação somativos [Silva e Silveira 2008,

\footnotetext{
${ }^{3}$ https://www.dropbox.com/s/jbxxjqz3e75k53l/ResultadosMapeamentoAvaliacaodeJogosomitido. $x l s x ? d l=0$ - Guia: Classificação por Avaliação.
} 
Hix e Hartson 1993]. Em relação aos resultados obtidos, todos eles apresentavam avaliações somativas, ou seja, avaliam protótipos ou o próprio JDE em sua versão final, muitas vezes já disponíveis em lojas de aplicativo.

\subsection{Contexto}

Para avaliação do contexto das avaliações dos JDEs apresentados pelos trabalhos obtidos, objetivou-se compreender quem eram os avaliadores (QS3). Dessa maneira, constatou-se que na grande maioria dos estudos, a avaliação foi realizada pelos próprios pesquisadores que, muitas vezes, também eram os desenvolvedores da ferramenta (46 artigos). Dentro desse montante, verificaram-se avaliações feitas em conjunto por especialistas e pesquisadores (artigos 138 e 170). Também houve trabalhos avaliados por educadores (artigos 178 e 264), sendo um deles em conjunto com especialistas (artigo 178).

No que tange à QS4, em relação ao contexto em que os jogos foram avaliados, foi identificado o público participante das avaliações. Devido à abordagem educacional, quase todas as avaliações envolveram o público-alvo dos jogos, os estudantes. Em relação à amostra, o mínimo de pessoas envolvidas nas avaliações em que os participantes eram especialistas, foram 2 (artigo 178). Em contrapartida, ao ter como participantes os estudantes ou usuários finais do jogo, a amostra mínima encontrada foi 8 (artigo 8) e o máximo 562 pessoas (artigo 406). Contando com os trabalhos em que foi informado a quantidade de participantes, a mediana de amostras é de 50, com idades que variam entre 4 e 55 anos.

Os estudantes participantes das avaliações foram classificados conforme a idade e/ou nível de escolaridade descritos pelos trabalhos. Todavia, devido às avaliações terem sido realizadas nos mais diversos países, adotou-se como base a subdivisão escolar brasileira que consiste em: Educação Infantil (de 0 a 6 anos) (6 artigos), Ensino Fundamental (de 7 a 14 anos) (21 artigos) e Ensino Médio (de 15 a 17 anos) (4 artigos). Também foram subdivididos os casos de trabalhos voltados ao Ensino Superior (15 artigos) e Ensino Especial (1 artigo). Além dos trabalhos em que os participantes eram apenas estudantes, encontrou-se outros contextos, como o caso em que participaram educadores e estudantes (artigo 227), apenas educadores (artigos 178 e 264), visitantes de um museu (artigo 300) e pessoas que falam alemão (artigo 394).

\subsection{Jogos sérios educacionais}

Em relação aos jogos sérios avaliados pelos artigos resultantes, identificou-se que $92 \%$ dos artigos apresentavam jogos gratuitos (44 artigos), sendo que desses, somente 7 estavam disponíveis para download, sugerindo um grande número de jogos acadêmicos. Todos os jogos comerciais dispunham de uma forma de acesso na web (4 artigos). Assim, 11 jogos estão disponíveis e 37 não estão disponíveis.

Um jogo foi avaliado em mais de um trabalho, sendo apresentado em 5 artigos diferentes. Dessa forma, o jogo Vidyanusa foi avaliado 4 vezes no ano de 2015 e 1 no ano de 2021 (artigos 127, 128, 227, 251 e 357). Ademais, em 6 artigos não havia a descrição dos nomes dos jogos avaliados (artigos 33, 82, 239, 413, 436 e 551). Em 5 artigos ocorreu a avaliação de mais de um jogo no estudo, sendo conjuntos com 3 (artigos 170, 391 e 406) e 4 jogos (artigos 269 e 430). Também em alguns trabalhos, plataformas de jogos foram avaliadas, como é o caso do Kahoot para criação de questionários em forma de 
jogo e The Smile and Learn que dispõem de vários jogos diferentes. Quanto ao foco do jogo, observou-se que a Taxonomia de Bloom [Bloom 1956] foi citada em 8 trabalhos diferentes como base para o desenvolvimento dos jogos educacionais. Isso demonstra o cuidado com o objetivo educativo desses jogos. Nesses trabalhos, foi possível analisar que 4 dos 6 objetivos educacionais provenientes da Taxonomia de Bloom são recorrentes, incentivando que o usuário entenda, aplique, analise e avalie o conteúdo fornecido pelo jogo educativo.

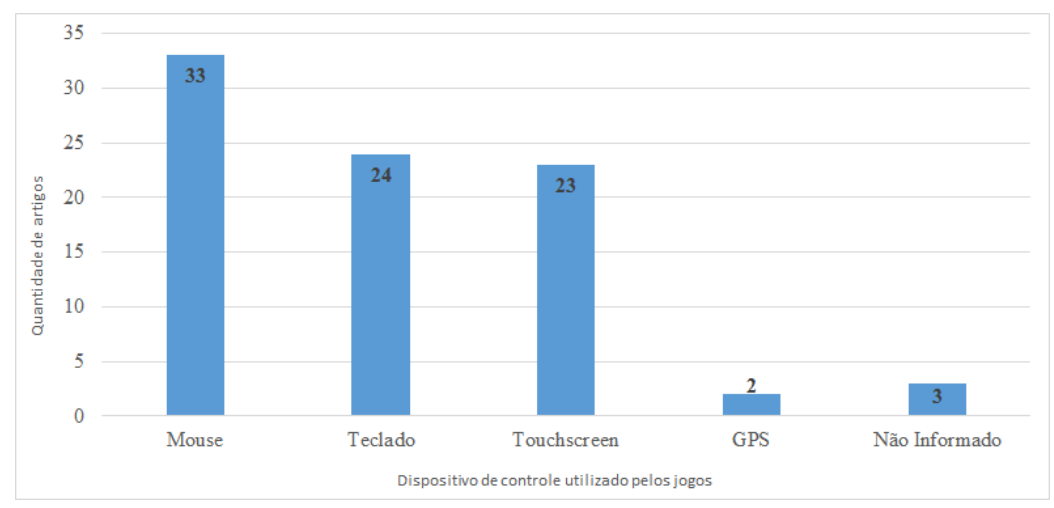

Figura 5. Dispositivos de Controle

Com base na QS5, foram obtidos os dados referentes aos dispositivos de controle usados nos jogos. Conforme apresentado na Figura 5, pode-se perceber que a grande maioria dos jogos usam o mouse como dispositivo de controle. Dentre os artigos avaliados, 17 deles apresentaram somente 1 dispositivo de controle, 3 não informaram (artigos 83, $430,553)$ e 28 trabalhos usaram mais de 1 dispositivo. Outra questão analisada são os jogos multiplataforma - jogos para diferentes sistemas operacionais -, assim, 12 trabalhos usaram jogos que trabalham com mouse e/ou teclado e também com o touchscreen e ainda, geolocalização como controle de jogo (artigos 300 e 637). Esses dados mostram que o uso de jogos para computadores ainda é frequente. Porém, essa é uma realidade em transformação, devido à disseminação dos dispositivos móveis e o acesso cada vez mais facilitado a crianças e jovens estudantes a smartphones e tablets, como mostrado na Seção 4.2 em que a maioria das avaliações ocorreram com um público na faixa etária dos 7 a 14 anos.

\section{Conclusão}

O objetivo deste estudo foi identificar na literatura trabalhos que apresentem a avaliação de jogos digitais sérios educacionais. Para isso, foi realizado um MSL para compreender como os JDEs são avaliados e a partir disso, realizar uma análise aprofundada sobre os métodos, o contexto e as características desses jogos. Como resultados, obtiveram-se 48 artigos pelos quais foi possível responder a questão de pesquisa e as questões secundárias estabelecidas para o mapeamento.

Quanto aos métodos utilizados (QS1), constatou-se o foco da grande maioria dos trabalhos em métodos de investigação, tendo como método mais utilizado, o questionário. Isso se dá pela facilidade de criar, aplicar e atingir um grande número de pessoas. A observação, segundo método mais utilizado, emprega o estudo de campo na grande maioria dos trabalhos, para realizar a avaliação no ambiente real, identificando assim, ações 
e reações mais fidedignas do público-alvo.

Ao verificar os métodos de avaliação, foi possível detectar os critérios/objetivos usualmente avaliados em JDEs (QS2), os quais se referem respectivamente a aprendizagem, usabilidade, experiência de usuário e engajamento do jogador. Da mesma maneira, verificou-se que ao realizar as avaliações, os autores dos trabalhos categorizam as informações de duas maneiras, dados quantitativos e dados qualitativos. Assim, nos trabalhos resultantes, obteve-se 45 quantitativos e 12 qualitativos. Observou-se que quem realiza as avaliações dos JDEs (QS3) são principalmente pesquisadores e ou desenvolvedores da ferramenta (46 artigos). Em relação ao contexto da avaliação (QS4), foi possível verificar um grande montante das participações sendo crianças estudantes do Ensino Fundamental, de 7 a 14 anos (21 artigos). Quanto aos controles usados (QS5), detectou-se uma proximidade entre os mais usados, sendo o mouse, o teclado e o touchscreen, demonstrando assim, que prevalece o desenvolvimento de JDEs para computadores de mesa e dispositivos móveis. Identificou-se também, controles atípicos como o GPS, o que demonstra a adaptação e atualização dos projetos com as novas tecnologias disponíveis (artigos 300 e 637).

Realizar o MSL possibilitou a constatação do estado da arte no que tange os JDEs avaliados no período de 2014 e 2021, permitindo compreender que os JDEs são uma ferramenta de ensino dinâmica e atrativa, capaz de gerar motivação e diversão aos estudantes. Além de que, podem ser aplicados aos mais diversos públicos, do ensino infantil ao ensino superior. Entretanto, devido ao seu contexto sério, inúmeras são as avaliações realizadas para garantir a qualidade do produto final. Com base na QP, identificou-se que os JDEs são avaliados considerando diversos critérios (usabilidade, experiência de jogador, aprendizagem, motivação, etc.) e inúmeros métodos. Desse modo, ao realizar as avaliações, os métodos podem ser usados em conjunto para análise de mais de uma característica do jogo, tornando a avaliação mais completa. Por conseguinte, essa é uma importante etapa do processo de desenvolvimento, a qual deve ser bem elaborada para garantir que o jogo cumpra com o seu objetivo dentro do ambiente escolar.

Como ameaça a validade pode-se considerar o entendimento subjetivo de cada avaliador sobre os critérios, entretanto para maior garantia da coerência de processo e dos dados extraídos, todos os artigos aceitos foram revisados por quatro pesquisadores participantes do estudo. Devido ao conjunto de MBAs utilizados, é passível que estudos relevantes não tenham sido incluídos. Os MBAs salvam as referências de forma diferente, e por isso pode ter ocorrido que ao importar para os softwares utilizados algum estudo não tenha sido incluído. Os resultados obtidos forneceram o embasamento para a tomada de decisões para a realização da avaliação de JDEs. Porém, como trabalhos futuros sugere-se a ampliação do MSL para outras bases de dados, maximizando a possibilidade de obter maiores informações sobre a fase de avaliação de jogos digitais sérios.

\section{Agradecimentos}

Os autores agradecem o apoio do Conselho Nacional de Desenvolvimento Científico e Tecnológico (CNPq) 308395/2020-4 e 313398/2019-4, FAPESC/CNPq No.06/2016 projeto T.O. 2017TR1755-Ambientes Inteligentes Educacionais com Integração de Técnicas de Learning Analytics e de Gamificação, FAPESC/UDESC No.04/2018 T.O 2019TR585 e FAPESC 27/2020-Apoio à Infraestrutura para Grupos de Pesquisa da Udesc. 


\section{Referências}

Anastasiadis, T., Lampropoulos, G., e Siakas, K. (2018). Digital game-based learning and serious games in education. International Journal of Advances in Scientific Research and Engineering, 4(12):139-144.

Barbosa, S. D. J., Silva, B. S. d., Silveira, M. S., Gasparini, I., Darin, T., e Barbosa, G. D. J. (2021). Interação Humano-Computador e Experiência do Usuário. Autopublicação.

Bloom, B. S. (1956). Taxonomy of educational objectives: The classification of educational goals by a committee of college and university examiners. McKay.

Buchinger, D., de Siqueira Cavalcanti, G. A., e da Silva Hounsell, M. (2014). Mecanismos de busca acadêmica: uma análise quantitativa. Revista Brasileira de Computação Aplicada, 6(1):108-120.

Cascini, D. e Campos, S. (2015). Avaliaçao de jogos educacionais multiusuários: Uma revisao sistemática da literatura. In Brazilian Symposium on Computers in Education (Simpósio Brasileiro de Informática na Educação-SBIE), volume 26, page 519.

Costa, A. L. B., Bonifácio, B. A., de Souza, B. P., e Fernandes, P. S. (2018). Applying teaching methods as requirements to develop games to assist children with learning disabilities: A case study. In 2018 XLIV Latin American Computer Conference (CLEI), pages 353-360. IEEE.

Derryberry, A. (2007). Serious games: online games for learning.

Grove, F. D., Bourgonjon, J., e Looy, J. V. (2012). Digital games in the classroom? a contextual approach to teachers' adoption intention of digital games in formal education. Computers in Human Behavior, 28(6):2023-2033.

Hix, D. e Hartson, H. R. (1993). Developing user interfaces: ensuring usability through product \& process. John Wiley \& Sons, Inc.

Nielsen, J. (1994). Heuristic evaluation. Usability inspection methods.

Peixoto, D. C. C., Resende, R. F., e Pádua, C. I. P. (2014). Evaluating software engineering simulation games: The ugalco framework. In 2014 IEEE Frontiers in Education Conference (FIE) Proceedings, pages 1-9.

Petersen, K., Feldt, R., Mujtaba, S., e Mattsson, M. (2008). Systematic mapping studies in software engineering. In 12th International Conference on Evaluation and Assessment in Software Engineering (EASE) 12, pages 1-10.

Prensky, M. (2003). Digital game-based learning. Computers in Entertainment (CIE), 1(1):21-21.

Prieto, L. M., Trevisan, M. d. C. B., Danezi, M. I., e Falkembach, G. M. (2005). Uso das tecnologias digitais em atividades didáticas nas séries iniciais. RENOTE-Revista Novas Tecnologias na Educação, 3(1).

Ramos, D. K., da Silva, G. A., e Macedo, C. C. (2020). Jogos digitais e emoções: um estudo exploratório com crianças. Revista Pedagógica, 22:1-21. 
Silva, T. S. e Silveira, M. S. (2008). Antecipando a avaliação de ihc: verificação de diretrizes a partir de modelos. In Proceedings of the VIII Brazilian Symposium on Human Factors in Computing Systems, pages 248-251.

Tarouco, L. M. R., Roland, L. C., Fabre, M.-C. J. M., e Konrath, M. L. P. (2004). Jogos educacionais. RENOTE: revista novas tecnologias na educação [recurso eletrônico]. Porto Alegre, $R S$.

Wangenheim, C. G. v., Barbosa, H., e Reolon, M. (2017). Heurísticas para avaliação de jogos educativos digitais - revisão sistemática de literatura. relatório técnico incod/gqs.05.2017.p.

Yuliana, I., Octavia, L. P., Sudarmilah, E., e Matahari, M. (2019). Introducing computational thinking concept learning in building cognitive capacity and character for elementary student. In 2019 19th International Symposium on Communications and Information Technologies (ISCIT), pages 549-554. IEEE. 